\title{
Normalisation of Neonatal Brain Network Measures Using Stochastic Approaches
}

\author{
Markus Schirmer ${ }^{1, \star}$, Gareth Ball ${ }^{1}$, Serena J. Counsell ${ }^{1}$, A. David Edwards ${ }^{1}$, \\ Daniel Rueckert ${ }^{2}$, Joseph V. Hajnal ${ }^{1}$, and Paul Aljabar ${ }^{1}$
}

1 Division of Imaging Sciences \& Biomedical Engineering, King's College London, UK

2 BioMedIA Group, Dept. of Computing, Imperial College London, UK

markus.schirmer@kcl.ac.uk

\begin{abstract}
Diffusion tensor imaging, tractography and the subsequent derivation of network measures are becoming an established approach in the exploration of brain connectivity. However, no gold standard exists in respect to how the brain should be parcellated and therefore a variety of atlas- and random-based parcellation methods are used. The resulting challenge of comparing graphs with differing numbers of nodes and uncertain node correspondences necessitates the use of normalisation schemes to enable meaningful intra- and inter-subject comparisons. This work proposes methods for normalising brain network measures using random graphs. We show that the normalised measures are locally stable over distinct random parcellations of the same subject and, applying it to a neonatal serial diffusion MRI data set, we demonstrate their potential in characterising changes in brain connectivity during early development.
\end{abstract}

Keywords: neonatal, MRI, diffusion, connectivity, network analysis.

\section{Introduction}

Over the last two decades, applications of network theory to a variety of fields have become more prevalent. Neuroimaging applications of network and graph theoretical techniques are comparatively recent (for an overview see e.g. [1]) and have demonstrated their utility in exploring the relations among regions in the brain and how these are affected by neural diseases [2]3].

In the brain, one often distinguishes between structural and functional connectivity. Functional connectivity is typically represented by correlations among the activations of different regions in the brain. By contrast, structural connectivity is based on estimation of neural pathways inferred from diffusion properties of cerebral white matter. It is possible to estimate this connectivity with diffusion tensor imaging [4. The work we present is generic and can be applied to any type of brain network data, although it will be described with a focus on structural connectivity in the developing neonatal brain.

Understanding the development and formation of structural connectivity after pre-term birth is highly desirable, as it has been shown that pre-term birth is

^ Corresponding author.

K. Mori et al. (Eds.): MICCAI 2013, Part I, LNCS 8149, pp. 574-581, 2013.

(C) Springer-Verlag Berlin Heidelberg 2013 
associated with adverse neurocognitive and behavioural outcome in later life [5]. Apart from practical difficulties in acquiring diffusion data from neonatal subjects, further challenges to neonatal connectivity analysis lie in the absence of an established set of regions with which to define the nodes of a network or graph. In adult data, network analysis of tractography data often relies on standard parcellations of the brain into a number of what are assumed to be functionally coherent regions. These define the nodes of a graph in which edges represent connected regions, which may be weighted by connection strength [6].

In neonates however, the lack of a standard set of regions has led to the adoption of approaches based on randomly generated parcellations of the brain as these rely on fewer assumptions regarding the presence of functionally defined regions. Ball et al. 8] apply a random parcellation approach to the cortical masks of a neonatal diffusion MRI (dMRI) dataset in a study of changes to cortical and thalamic connectivity in preterm infants. In their work, Poisson disk sampling 9] was used to generate around 500 region centres with a constraint on the minimum distance between each pair. In a tractography analysis streamlines were propagated from every region and the connection strength between the thalamus and each target region was calculated as integrated anisotropy along the path 811. Streamlines connecting a region with itself were removed. Repeating this for different random parcellations generates multiple connectivity matrices for a single subject, which provide per-node network measures that can be propagated back to provide voxel-wise maps.

Challenges of Stochastic Parcellations. Defining the regions stochastically means that the number of regions can vary across parcellations of the same brain. For example, Poisson disk sampling specifies a target number of regions but this can vary from the number of regions generated due to the distance constraint. It is also important to note that some network measures have been shown to be highly dependent on the number of regions [12].

This motivates the need to address potential biases in network measures derived from randomised parcellations of dMRI data. It is important both for generating summary measures for an individual brain from multiple connectivity matrices and for making meaningful inter- and intra-subject comparisons of network-based measures, particularly across different studies. The dependence of measures on the number of regions/nodes is illustrated in Fig. 1 for a particular network measure (clustering coefficient) obtained from a set of connectivity matrices based on random parcellations of the same subject.

This work presents techniques for removing the number of region dependence from network measures. Our methods normalise a measure $m(G)$ on a directed and weighted graph $G$ against the value of $m\left(G^{\prime}\right)$, where $G^{\prime}$ is obtained by randomly perturbing the structure of $G$, by modifying its edge structure and/or edge weights. These randomised networks may not represent physiologically plausible networks, however, they may be seen as 'white noise' used to identify the significance and change of network measures obtained from imaging data. The process is schematically illustrated in Fig. 2. We show that established random graph approaches can yield unsuitable normalisations for measures such as the 

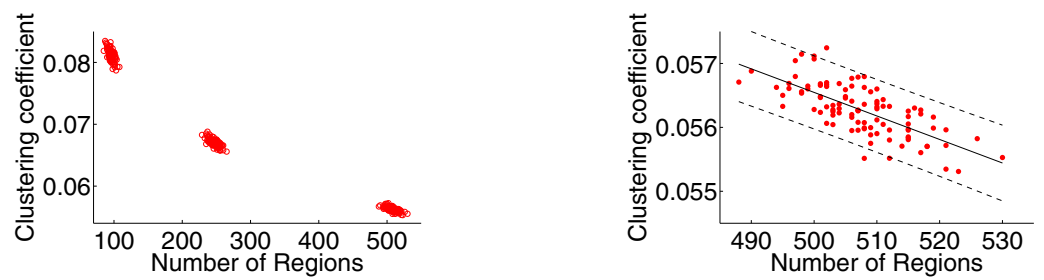

Fig. 1. The dependence of the clustering coefficient on the number of regions in different parcellations of the same subject. Left: Sets of parcellations with around 100, 250 and 500 regions. Right: Detailed view around 500 regions.

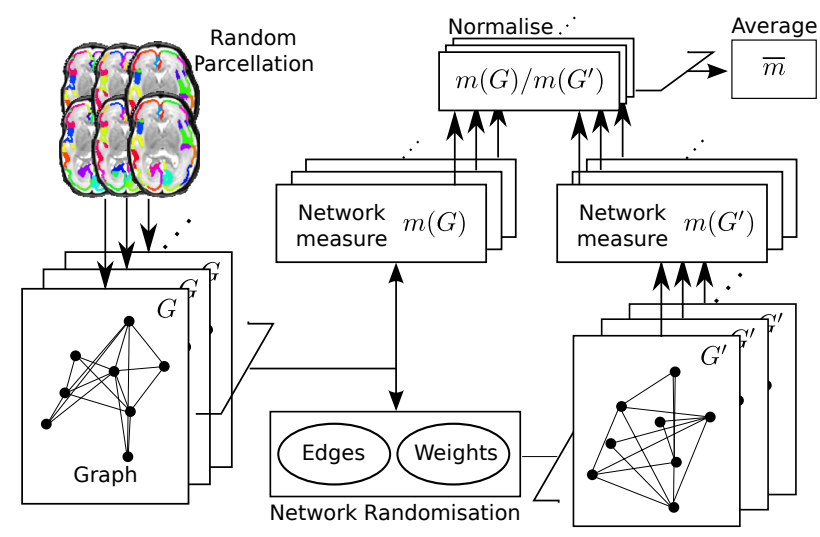

Fig. 2. Processing pipeline used for removing the number of region dependence

clustering coefficient and present a novel approach which allows for a better comparison. Furthermore, we validate our method by applying it to serial dMRI data of 16 neonates and show the evolution of their network measures over time.

\section{Methods and Materials}

The methods for generating a random realisation of a graph obtained from the random parcellations of the cortical mask are described below. We emphasise the distinction of the stochastic step for generating a random network during normalisation and the one used to generate the initial parcellations on which an observed network is defined.

Edge Structure. Two established algorithms to create random networks for comparison use either an Erdös-Rényi model (ER) or pairwise switching of the edges (PS), an algorithm which keeps the number of outgoing edges of each node constant. In this section we will briefly review both approaches. Not altering the edge structure will be referred to as edge preserving (EP). 
We denote a graph $G=\{V, E, W\}$, where $V$ and $E$ are the vertex and edge sets and $W=\left\{w_{i j}\right\}$ the set of edge weights, which represent the connectivity matrix derived from the tractography. We denote the neighbourhood $N_{i}$ of node $i$ to be the set of edge-wise neighbours $\{j \in V: i j \in E\}$ and $G$ is assumed to be simple, i.e. without multiple edges or self-loops.

For $P S$, nodes $r$ and $s$ are randomly selected from $V$ and $t$ and $u$ are randomly chosen from $N_{r}$ and $N_{s}$ respectively. If the graph remains simple, remove the edge $r t$ and $s u$ from $E$ and add $r u$ and $s t$ [13. In order to amplify the perturbation of the graph, we modified PS to maximise its effect. For each node $v \in V$, we iterate over all nodes $r \in N_{v}$, select $s \in V \backslash\{r\}$ and apply PS to $r$ and $s$. This is repeated for all nodes. By contrast, $E R$ starts with the same number of nodes $N$ as the observed graph. Edges are then randomly assigned to node pairs until the number of edges equals the number in the observed graph.

Weight Assignment. The white matter connectivity networks studied are weighted by the integrated anisotropy, necessitating a method for assigning weights to the edges of the derived random graphs. We applied three types of methods for achieving this: The original weights set may be redistributed naturally in case of PS by switching them along with the edges, or randomly permuted in EP/ER. We also draw weights uniformly from the interval $[0, \max ]$, where max is the maximum weight in the original graph. Finally we draw weights at random from the weight set based on the shortest path distances between all node pairs in the original graph, which are calculated using the Dijkstra algorithm. For the last method, distances along each edge $i j$ are converted as $1 / w_{i j}$. Subsequently calculated shortest distances between all node pairs are then converted back to weights, again as reciprocals, to generate a complete weighted graph matrix. The entries in this matrix are then drawn for assignment to the perturbed graph.

Network Measures. There is a vast literature on measures for characterising networks. A good summary of measures commonly used for brain networks is given by Rubinov and Sporns [14].

Betweenness centrality (BC) relates to the amount of information passing through a particular node, assuming that information travels along the shortest paths. A node $s$ in a graph has $B C(s)$ given by $B C(s)=\sum_{r, t: r \neq s \neq t} \frac{\lambda_{r t}(s)}{\lambda_{r t}}$, where $\lambda_{r t}$ is the number of shortest paths from $r$ to $t$ and $\lambda_{r t}(s)$ is the number that pass through $s$. The BC of the network can be characterised by the mean of $B C(s)$ over all nodes.

The out-degree $k_{\text {out }}(s)$ of a node $s$ in a weighted network is defined as the sum of the weights of all outgoing connections. It represents a measure of how well a node is connected within a network. We calculated the mean out-degree, $k_{\text {out }}$, over all nodes in order to compare this measure across networks.

The clustering coefficient, $C(s)$ of a node $s$, is a commonly used measure which represents the ratio of triangles containing $s$ to the maximum number possible. $C(s)$ is given by [15]

$$
C(s)=\frac{t_{s}}{k(s)(k(s)-1)-2 \sum_{r \in V} e_{s r} e_{r s}},
$$


where $t_{s}$ is the number of directed triangles around node $s, k(s)=k_{\text {out }}(s)+$ $k_{i n}(s)$ is the total degree and $e_{i j}=1$, if $i j \in E$ and zero otherwise. The clustering coefficient $C$ of a network can then be calculated as the average of $C(s)$ over all nodes $s$. It can be interpreted as the predominance of clustered components around nodes. It is also used in determining small-worldness, as small-world networks tend to have higher clustering coefficients.

The characteristic path-length, $\lambda(s)$, of a node $s$ is defined as average shortest path-length between $s$ and all other nodes in the network. It can be interpreted as a measure of integration of a particular node in the network and is given by $\lambda(s)=\frac{1}{|V|-1} \sum_{t \neq s} d_{s t}$, where $d_{s t}$ is the shortest distance between nodes $s, t \in V$ and $|V|$ is the total number of nodes. The mean measure, $\lambda$, is referred to as global characteristic path-length. It also relates to small-worldness, as smallworld networks tend to have lower values of $\lambda$.

The efficiency $E(s)$ of node $s$ is computed similarly to the characteristic pathlength. In this case, however, the average is taken over the reciprocals of the shortest paths. The advantage of the efficiency over the characteristic pathlength lies in the meaningful computation for multi-component networks, as infinite paths between nodes in disconnected components have zero efficiency. The overall efficiency of a network can be expressed by the mean of $E(s)$ over all nodes $s$, where $E(s)$ is given by $E(s)=\frac{1}{|V|-1} \sum_{t \neq s} d_{s t}^{-1}$.

The diameter of a network is given by the longest of all the shortest paths in the network and is representative of the size of the network. In case of information flow it can therefore represent the longest path (or time) for a signal to flow from any node to any other node.

Data. Serial dMRI data was acquired from 16 infants born at less than 32 weeks gestational age and recruited as part of ongoing studies at Queen Charlotte's and Chelsea Hospital. Mean gestational age at birth was $28.0 \pm 2.4$ weeks and the mean age at scan was $30.8 \pm 2.1$ and $41.1 \pm 1.0$ weeks at the first and second scans respectively. Each infant successfully underwent 32-direction dMRI. Single shot echo planar imaging dMRI was acquired in the transverse plane in 32-non-collinear directions using the following parameters: TR: $8000 \mathrm{msec}$;E: 49 msec; slice thickness: $2 \mathrm{~mm}$; field-of-view: $224 \mathrm{~mm}$; matrix: $128 \times 128$ (voxel size: $1.75 \times 1.75 \times 2 \mathrm{~mm}$; b-value: $750 \mathrm{sec} / \mathrm{mm}^{2}$; SENSE factor of 2 . Random cortical parcellations were obtained using Poisson disk sampling [9] and a probabilistic diffusion tractography algorithm was applied [10. Diffusion MRI data were pre-processed using the FSL's Diffusion Toolkit and corrected for geometric distortions. For more details the reader is referred to [8].

\section{Results}

In a series of experiments, we assessed the performance of the different normalisation schemes, each consisting of a method for determining edge structure and a method of assigning edge weights as described above. The results of comparing the different schemes for parcellations with around 500 regions are summarised in Table 1. The variance explained by the number of regions was used to assess 
each measure and the mean explained variance over 12 runs for each of 8 different subjects was recorded for each measure. On average, the best performance (largest reduction in explained variance) was obtained by schemes that drew the weights of the randomised graph uniformly from an interval between zero and the maximum weight of the original matrix. In particular, Table 1 shows that the established ER and PS methods, with weights drawn from the original graph, performed badly with respect to the clustering coefficient. The approach based on Dijkstra performed particularly poorly with respect to the clustering coefficient and introduced a higher out-degree correlation than originally present. We will therefore focus further analyses on the subcategory of altering the edge weights with the uniformly drawn weights.

Table 1. The variance (\%) of each measure explained by the number of regions in a network before and after each normalisation scheme (original weights $(\mathrm{OW})$, uniform (UNI), Dijkstra (D), Erdös-Rényi (ER), pairwise switching (PS), edge preserving (EP)) for all measures. Stars mark the lowest value for each measure. In case of the missing values $(\mathrm{OW})$ the measures stayed the same after normalisation.

\begin{tabular}{|c|c|c|c|c|c|c|c|c|c|c|}
\hline & & \multicolumn{3}{|c|}{ ER } & \multicolumn{3}{c|}{ PS } & \multicolumn{3}{c|}{ EP } \\
\hline & Before & OW & UNI & D & OW & UNI & D & OW & UNI & D \\
\hline$B C$ & 76.7 & 10.1 & 2.9 & 9.6 & 10.8 & 2.6 & 5.6 & - & 2.7 & $1.1^{*}$ \\
\hline$k_{\text {out }}$ & 7.8 & - & $2.2^{*}$ & 33.3 & - & $2.2^{*}$ & 33.6 & - & $2.2^{*}$ & 34.0 \\
\hline$C$ & 49.3 & 43.4 & 7.7 & 45.1 & 42.7 & 3.8 & 52.9 & - & $2.2^{*}$ & 32.5 \\
\hline$\lambda$ & 28.6 & 11.4 & 2.2 & 8.1 & 7.7 & 2.1 & 10.1 & $0.7^{*}$ & 2.1 & 21.2 \\
\hline$E$ & 36.0 & 12.3 & 2.2 & 18.0 & 6.6 & 2.2 & 22.5 & $1.0^{*}$ & 2.2 & 32.0 \\
\hline dia & 2.8 & 2.0 & 2.1 & 1.2 & 1.9 & 1.7 & 1.2 & - & $1.1^{*}$ & 0.8 \\
\hline
\end{tabular}

In order to decide on the edge structure method, we considered their performance over a wider range of regions. For this, we parcellated a data set with target numbers of 100, 250 and 500 regions, creating 100 networks in each case. This analysis showed that EP outperformed ER and MPS by reducing the explained variance on average the most for both the 100 and 250 region parcellations.

Based on these results, we selected the combination of EP with uniformly drawn weights. We applied this scheme to the serial dMRI data of all subjects with around 500 region parcellations by generating one random realisation of the observed network for normalisation. Repeating the process multiple times did not yield major improvements. Figure 3 shows the change over time of the resulting normalised measures. We assessed the normalised measures in terms of the consistency of their change with time. In almost all the cases, the clustering coefficient increases with age at scan, while the characteristic path-length decreases. This suggests that the degree to which the brain represents a smallworld network increases with age. The out-degree and efficiency both increase with time, indicating improved connectivity in the brain with age. The results for $\mathrm{BC}$ and network diameter are not conclusive, showing no consistent change over time. However, the average betweenness centrality is consistently much lower than in each corresponding random network $(\approx 0.22)$. This suggests an 
increased resilience to node failure or 'attacks' on the network. We performed paired t-tests for the normalised serial measures of all subjects. These were significant $(p<0.001)$ for all measures, except for $\mathrm{BC}$ and diameter.
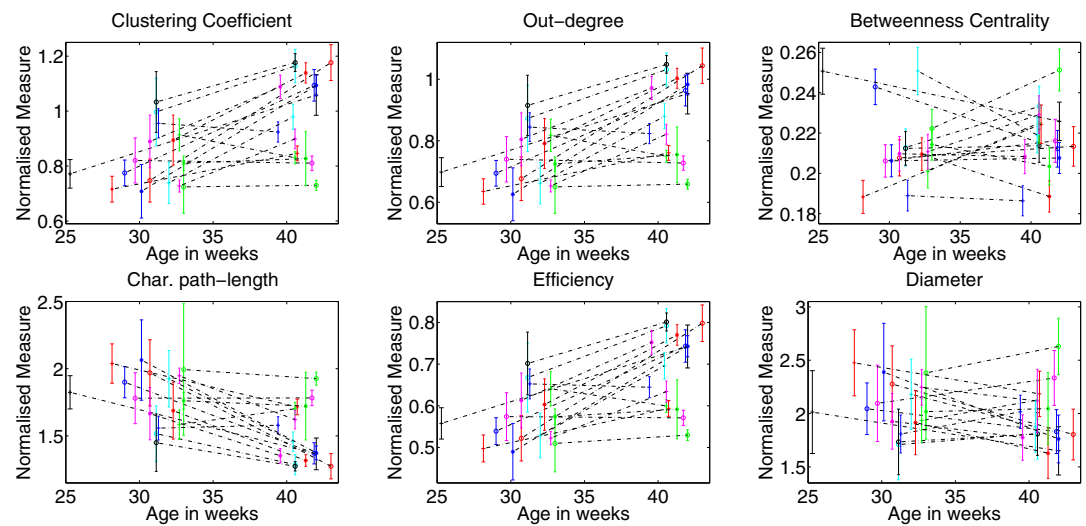

Fig. 3. Median normalised values of network measures determined from 100 parcellations per subject. Each colour-marker combination represents one infant with the distribution of measures from multiple randomly parcellated networks at two time points (connected by a dashed line). Four of six measures show a distinct tendency in their evolution in time (see text).

\section{Conclusion}

We presented novel approaches for generating random graphs from neural connectivity graphs for the purpose of normalising network measures. The results indicate that edge preserving with uniformly drawn weights between zero and the maximum of the original weights outperforms established approaches. Although some dependence on the number of regions remained over the larger scale of 100500 regions, it allowed for a better local comparison of measures for networks with around 500 regions. It should be noted that, with fewer regions, there was greater variation in both the normalised and raw network measures. This highlights the difficulty of comparing network measures across the different scales that are commonly used in atlas-based methods [16]. In future work, we will focus on eliminating the number of region dependence on a wider scale and on determining the optimal number of regions for representing brain networks. We note that the benefit of the presented random graph approach over regressionbased models is that only one observed network is required to carry out the normalisation, while a number of networks would be needed for regression. In comparison with trend analysis, our method therefore provides a comparatively cheap approach for normalising against the number of regions and it enables network measures to be compared across subjects and over time. 
Acknowledgments. This research was supported by the National Institute for Health Research (NIHR) Biomedical Research Centre at Guy's and St Thomas' NHS Foundation Trust and King's College London. The views expressed are those of the author(s) and not necessarily those of the NHS, the NIHR or the Department of Health. Medical Research Council (MRC) Centre for Transplantation, King's College London, UK — MRC grant no. MR/J006742/1.

\section{References}

1. Sporns, O.: The human connectome: a complex network. Ann. N.Y. Acad. Sci. 1224, 109-125 (2011)

2. Bullmore, E., Sporns, O.: Complex brain networks: graph theoretical analysis of structural and functional systems. Nat. Rev. Neurosci. 10(3), 186-198 (2009)

3. Supekar, et al.: Network analysis of intrinsic functional brain connectivity in Alzheimer's disease. PLoS Comput. Biol. 4(6), 1-11 (2008)

4. Alexander, et al.: Diffusion tensor imaging of the brain. Neurotherapeutics 4(3), 316-329 (2007)

5. Delobel-Ayoub, et al.: Behavioral problems and cognitive performance at 5 years of age after very preterm birth: the EPIPAGE Study. Pediatrics 123(6), 1485-1492 (2009)

6. Sporns, O., Tononi, G., Kötter, R.: The human connectome: A structural description of the human brain. PLoS Comput. Biol. 1(4), 245-251 (2005)

7. Hagmann, et al: Mapping the structural core of human cerebral cortex. PLoS Biol. 6(7), 1479-1493 (2008)

8. Vall, et al.: The influence of preterm birth on the developing thalamocortical connectome. Cortex, 1-11 (2012)

9. Bridson, R.: Fast poisson disk sampling in arbitrary dimensions. In: ACM SIGGRAPH, vol. 2007 (2007)

10. Behrens, et al.: Probabilistic diffusion tractography with multiple fibre orientations: What can we gain? Neuroimage 34(1), 144-155 (2007)

11. Robinson, et al.: Identifying population differences in whole-brain structural networks: a machine learning approach. NeuroImage 50(3), 910-919 (2010)

12. Zalesky, et al.: Whole-brain anatomical networks: does the choice of nodes matter? NeuroImage 50(3), 970-983 (2010)

13. Maslov, S., Sneppen, K.: Specificity and stability in topology of protein networks. Science 296(5569), 910-913 (2002)

14. Rubinov, M., Sporns, O.: Complex network measures of brain connectivity: uses and interpretations. NeuroImage 52(3), 1059-1069 (2010)

15. Fagiolo, G.: Clustering in complex directed networks. Phys. Rev. E 76(2), 026107 (2007)

16. Van Wijk, et al.: Comparing brain networks of different size and connectivity density using graph theory. PloS one 5(10), 13701 (2010) 